\title{
Advances in Lasers and Optical Amplifiers: Materials, Components, and Systems
}

\author{
Francesco Prudenzano, ${ }^{1}$ Frédéric Smektala, ${ }^{2}$ and Luciano Mescia ${ }^{1}$ \\ ${ }^{1}$ Dipartimento di Ingegneria Elettrica e dell'Informazione (DIEI), Politecnico di Bari, Via E. Orabona 4, 70125 Bari, Italy \\ ${ }^{2}$ Laboratoire Interdisciplinaire Carnot de Bourgogne (ICB), UMR 6303 CNRS-Université de Bourgogne, 9 Avenue, Alain Savary, BP \\ 47870, 21078 Dijon, France
}

Correspondence should be addressed to Francesco Prudenzano, prudenzano@poliba.it

Received 6 November 2012; Accepted 6 November 2012

Copyright (C) 2012 Francesco Prudenzano et al. This is an open access article distributed under the Creative Commons Attribution License, which permits unrestricted use, distribution, and reproduction in any medium, provided the original work is properly cited.

During the last decades a lot of research activities in the field of photonics have been accomplished and today there is a disruptive revolution underway where the photons are used to transmit, store, and process information. Laser and optical amplifier technology plays an important role in these events. In particular, the advances in lasers and optical amplifiers, enabling novel, powerful and exciting applications are based on research results arising from different investigation fields. This special issue is an example of these interdisciplinary contributions to the state of the art of optical amplification. Microresonators, interferometry, accurate modeling, grating, nonlinear optics, and novel materials are the subjects included in the volume.

In the paper entitled "Modeling of mid-IR amplifier based on an erbium-doped chalcogenide microsphere" the authors illustrate the design of an optical amplifier based on a tapered fiber and an $\mathrm{Er}^{3+}$-doped chalcogenide microsphere. The obtained simulation results demonstrate that a highefficiency mid-IR amplification can be obtained by using a quite small microsphere. The low threshold power and the high gain in the mid-IR suggest interesting application in sensing.

The paper "Light combining for interferometric switching" describes a novel switching device based on three input phase modulation ports. The interferometric switching as a routing method in sequence of coupled optical microresonators is investigated. Extended appendices facilitate the explanation of the implemented model. The proposed interferometer device shows the possibility to control a lightwave signal with the phase of another signal with interesting and feasible applications.
The paper "Detailed theoretical model for adjustable gainclamped semiconductor optical amplifier" depicts the modeling and the experimental characterization of an adjustable gain-clamped semiconductor optical amplifier (AGC-SOA). The simulations agree very well with the experimental results. The proposed device could operate as power equalizer and linear amplifier in packet-based dynamic systems such as passive optical networks (PONs).

"High-energy passive mode-locking of fiber lasers" is a comprehensive study of achieving high-energy pulses in a ring cavity fiber laser, passively mode-locked by a series of waveplates and a polarizer. The obtained results may be used as practical guidelines for designing high-power lasers.

In the paper "Tunable single-longitudinal-mode highpower fiber laser" the authors show a novel CW tunable, highpower, single-longitudinal-mode fiber laser. A tunable fiber Bragg grating for wavelength selection is exploited. Due to the narrow linewidth and high output power, this laser could find applications in developing sensor based on nonlinear effects such as stimulated Brillouin scattering.

The paper "New trends in amplifiers and sources via chalcogenide photonic crystal fibers" is a review on amplification and lasing via rare earth doped chalcogenide photonic crystal fibers. Materials, devices, and feasible applications in the mid-IR are mentioned.

\section{Acknowledgments}

The guest editors are pleased and honored to submit this special issue to Hindawi Publishing Corporation and 
thank all the authors and reviewers for their valuable contribution.

Francesco Prudenzano Frédéric Smektala

Luciano Mescia 

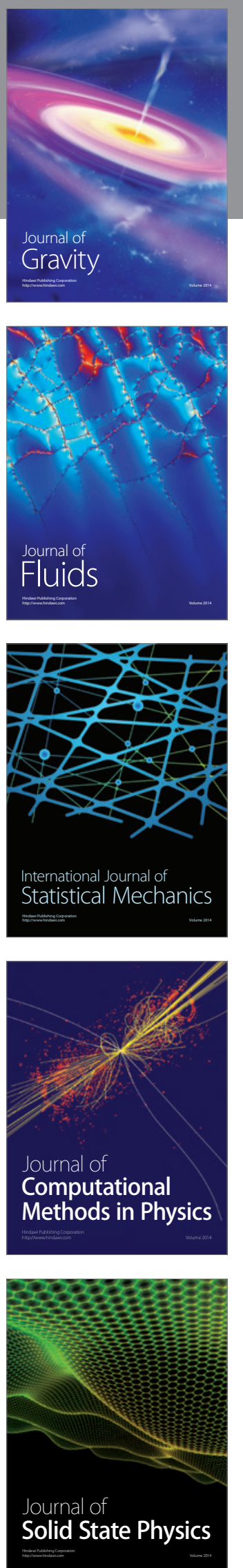

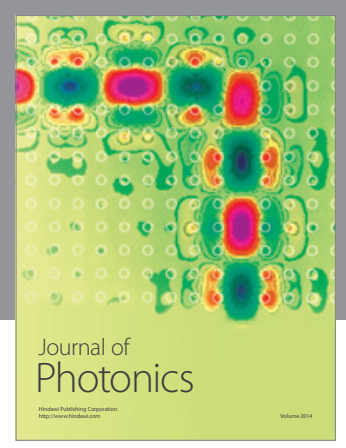

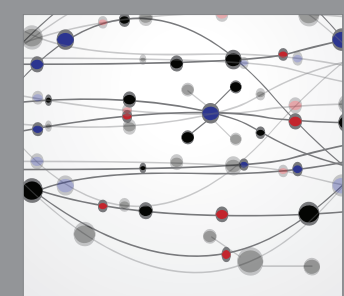

The Scientific World Journal
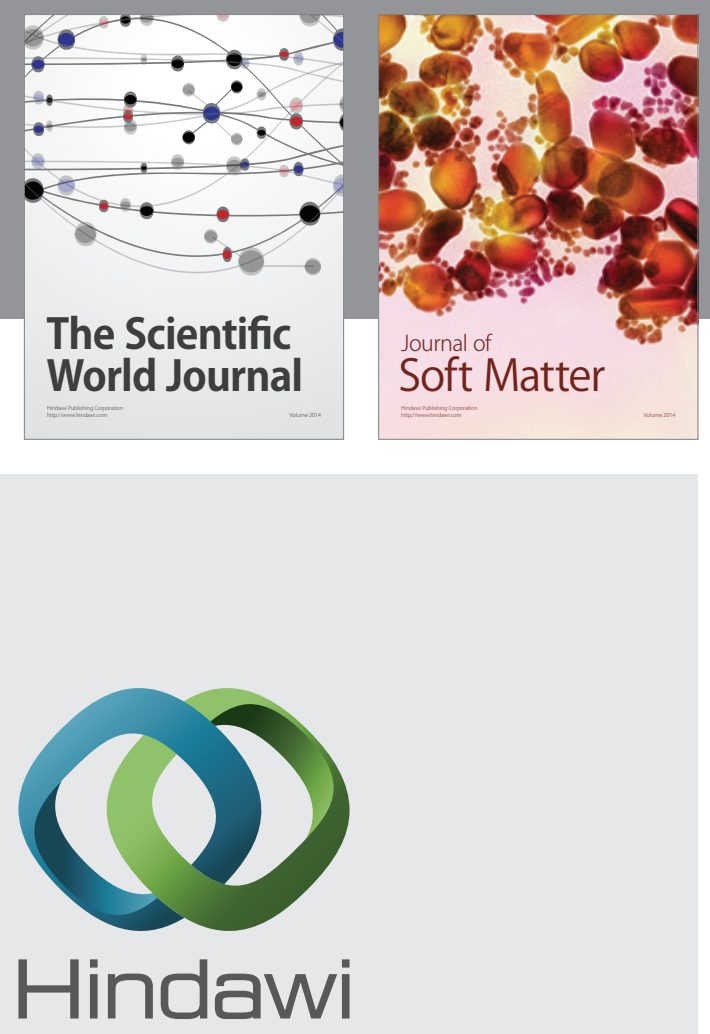

Submit your manuscripts at

http://www.hindawi.com
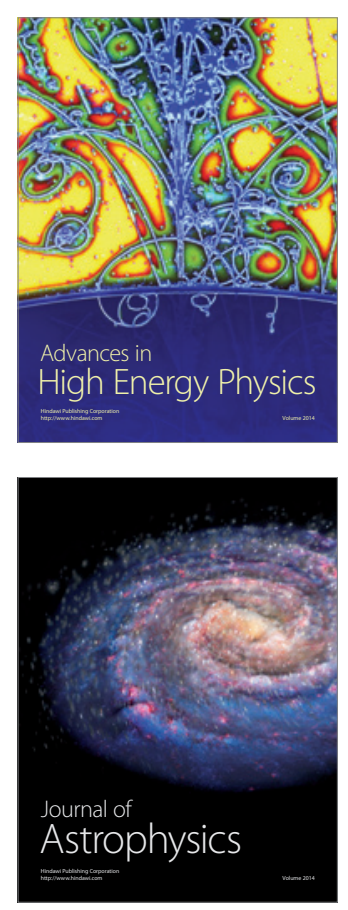
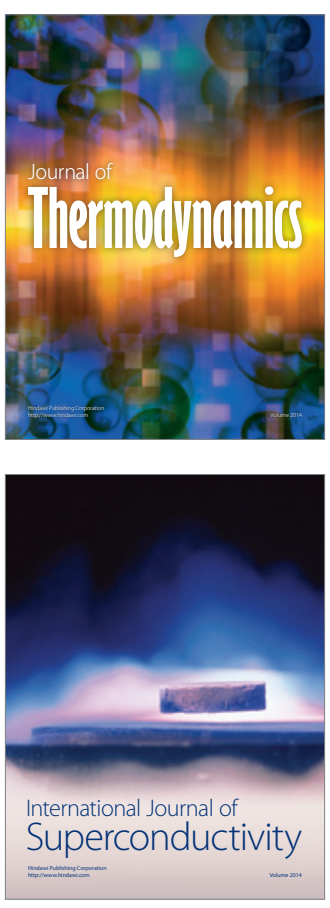
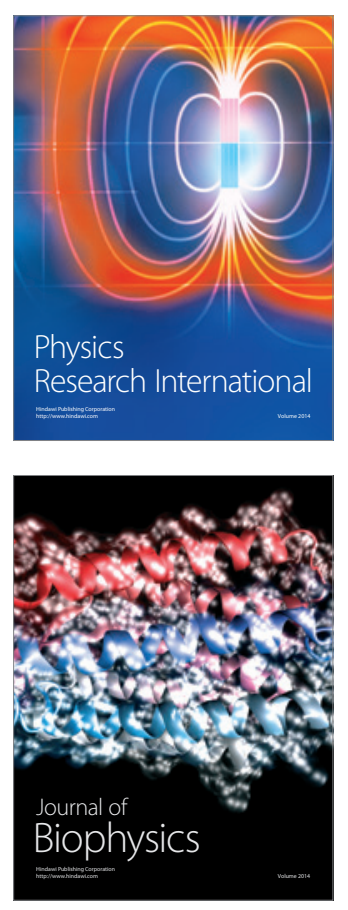
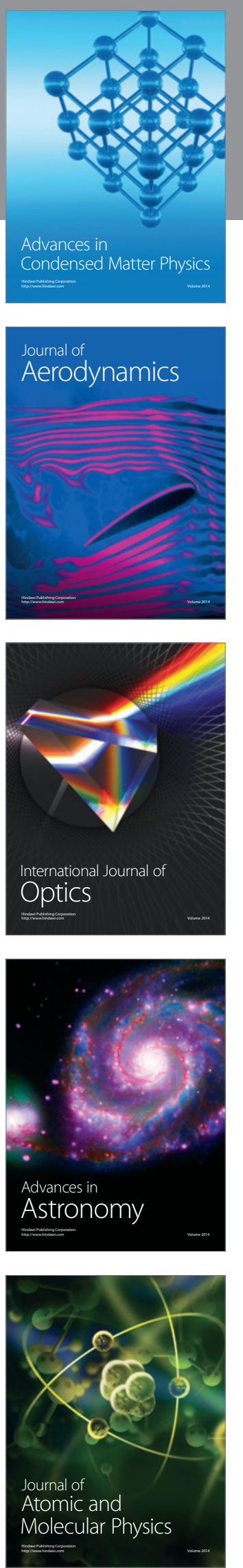